\title{
Registro de petróleo em poríferos e cnidários durante o impacto agudo de derramamento no Nordeste brasileiro em 2019
}

Oil record in sponges and cnidarians during the acute spill impact in northeastern Brazil in 2019

\author{
W. R. P. Cerqueira ${ }^{1 *}$; R. N. Batista ${ }^{1}$; V. O. $\operatorname{Santos}^{1}$; J. L. Barbarino²; G. S. \\ Quaglio $^{3}$; P. H. S. M. Reis ${ }^{3}$ \\ ${ }^{I}$ Departamento de Ciências Biológicas/Museu de Zoologia/ Divisão de Invertebrados Aquáticos, Universidade \\ Estadual de Feira de Santana, 44036-900, Feira de Santana-Bahia, Brasil \\ ${ }^{2}$ Departamento de Ciências Biológicas/ Laboratório de Taxonomia Vegetal, Universidade Estadual de Feira de \\ Santana, 44036-900, Feira de Santana-Bahia, Brasil \\ ${ }^{3}$ Departamento de Ciências Biológicas, Universidade Estadual de Feira de Santana, 44036-900, Feira de Santana- \\ Bahia, Brasil \\ *walter@uefs.br
}

(Recebido em 26 de março de 2020; aceito em 12 de agosto de 2020)

\begin{abstract}
Toneladas de petróleo atingiram centenas de localidades ao longo da costa nordestina brasileira entre agosto e dezembro de 2019. A mídia enfatizou o impacto apenas para os vertebrados de grande porte ou com apelo midiático, e algumas pesquisas acadêmicas foram feitas com foco em espécies de interesse comercial, não contemplando outras espécies que também podem ter sido afetadas, em especial os invertebrados bentônicos sésseis ou de pouca mobilidade. Este trabalho teve como objetivos conferir a existência de resquícios de óleo em uma praia urbana da cidade após a limpeza feita por voluntários e funcionários de órgãos governamentais e investigar a ocorrência de petróleo em algumas espécies de poríferos e cnidários comuns nos afloramentos rochosos do litoral de Salvador. Após a limpeza feita por voluntários e órgãos governamentais. Os exemplares coletados foram identificados e necropsiados sob lupa. Como resultado das observações de campo verificouse que, mesmo após a limpeza, a praia continuava apresentando diversos vestígios de óleo na areia, nas rochas e também nas algas. Foram identificadas quatro espécies de Porifera (Amphimedon viridis, Cinachyrella apion, Cliona varians e Desmapsamma anchorata) e quatro de Cnidaria (Bellactis ilkaliaseae, Bunodosoma cangicum, Palythoa caribaeorum e Zoanthus sociatus), sendo encontrados vestígios de petróleo na espongiocele de $D$. anchorata e na faringe de $B$. ilkaliaseae. Tais registros ampliam o número de taxa afetados pelo impacto agudo após o evento de derramamento de óleo no Nordeste em 2019.
\end{abstract}

Palavras-chave: hidrocarbonetos, poluição, bentos.

Tons of oil reaching hundreds of locations along the Brazilian northeastern coast between August and December 2019. The news emphasized the impact only for large vertebrates or those with media appeal, and some academic research was done with a focus on commercial interest, disregarding how the oil may be affecting hundreds of other species, especially the sessile or poorly mobile benthic invertebrates. This paper aimed to investigate the occurrence of oil in some sponges and cnidarians species usual in beach rocks of Salvador and to verify the presence of oil residues on an urban beach in the city after cleaning by workers and volunteers. The collected specimens were identified and necropsied under a magnifying glass. As a result of the changes in the field that occurred after cleaning, the beach continues to show several traces of oil in the sand, rocks, and algae. Were identified four species of Porifera (Amphimedon viridis, Cinachyrella apion, Cliona varians, and Desmapsamma anchorata) and four of Cnidaria (Bellactis ilkaliaseae, Bunodosoma cangicum, Palythoa caribaeorum and Zoanthus sociatus). Traces of oil were found in the spongiocele of $D$. anchorata and in the pharynx of $B$. ilkaliaseae. These records increase the number of taxa affected by the impact after the oil spill event in the Northeast of 2019.

Keywords: hydrocarbons, pollution, benthos.

\section{INTRODUÇÃO}

Eventos de mortalidade em massa de organismos bentônicos durante e após impactos agudos de derramamento de petróleo são conhecidos desde a expansão da prospecção de combustíveis fósseis nos ambientes marinhos, onde em algumas horas após um acidente já são observados indivíduos 
mortos pertencentes a diversos grupos taxonômicos [1]. O petróleo é fatal para os organismos aquáticos em função de seu potencial toxicológico que afeta processos metabólicos (crescimento e biomassa) e genéticos (mutações), além de matar por asfixia após o óleo obstruir mecanicamente estruturas como as brânquias [2,3]. O óleo afeta os organismos marinhos também em seus aspectos ecológicos, ao desestruturar as cadeias tróficas [3].

As esponjas constituem um importante elemento das comunidades bentônicas dos ambientes marinhos, onde ocorrem em altas densidades desde a zona entre marés até o mar profundo, servindo como hábitat para uma gama de invertebrados crípticos que ocupam seus poros para se refugiar de predadores e se alimentar do plâncton e matéria orgânica particulada presentes na água que entra pelos óstios [4]. Diversos animais marinhos predam as esponjas, a exemplo de peixes, tarturugas e equinodermos. A utilização desses animais sésseis como fonte de alimento amplia ainda mais a necessidade de estudos que demonstrem o impacto do derramamento de óleo nas comunidades de esponjas, já que, através da predação, o óleo pode vir a alcançar níveis tróficos superiores [34, 35, 37]. Alguns impactos que um derramamento de óleo pode provocar sobre as comunidades de esponjas seriam as alterações na sua microbiota, mortalidade das larvas e o entupimento de seus poros através da ação mecânica, afetando não apenas estes animais, mas todos os outros que os habitam como inquilinos $[4,5]$.

Assim como os poríferos, os cnidários, a exemplo das anêmonas-do-mar, desempenham um papel importante no fluxo de energia das cadeias tróficas dos ecossistemas marinhos, atuando como carnívoros [6]. Este filo de invertebrados é bastante estudado por pesquisadores da área de saúde pois as suas toxinas podem provocar severos acidentes em humanos [7]. Algumas formas que o petróleo bruto e os hidrocarbonetos policíclicos aromáticos (HPAs) podem afetar as anêmonas do mar e outros cnidários consistem em alterações na expressão gênica, atividade enzimática, mortalidade de larvas e de recrutas [8].

De agosto a dezembro do ano de 2019 a região Nordeste do Brasil conviveu com o maior impacto agudo de derramamento de petróleo da sua história. Em 3 meses (final de agosto a início de novembro) toneladas de óleo cru altamente tóxico, de origem ainda desconhecida, se espalharam por cerca de $70 \%$ dos $3.300 \mathrm{~km}$ da costa nordestina, provocando um enorme impacto não só na biodiversidade, mas também com prejuízos socioeconômicos e da saúde da população $[9,10]$.

Estudos conduzidos durante o impacto agudo registraram a presença de petróleo nos tratos respiratório e digestivo de espécies de importância pesqueira como peixes, moluscos e crustáceos [10], ficando de fora das análises os invertebrados bentônicos sem interesse comercial, embora estes tenham importância ecológica inclusive para incrementar a produtividade pesqueira nas áreas afetadas. Apesar de muitos invertebrados marinhos a exemplo das esponjas e cnidários atuarem como elementos chave para a manutenção da biodiversidade marinha, apenas as "espécies midiáticas" como tartarugas, golfinhos e aves são alvo de comoção nas redes sociais e noticiadas pela imprensa como se fossem as únicas a sofrer com impactos ambientais.

Tendo em vista os fatos apresentados e a necessidade de produzir conhecimentos acerca do derramamento agudo de óleo no Nordeste no ano de 2019, este trabalho teve como objetivos: 1 conferir a existência de resquícios de óleo em uma praia urbana da cidade após a limpeza feita por voluntários e funcionários de órgãos governamentais e, 2 - investigar a ocorrência de petróleo em algumas espécies de poríferos e cnidários comuns nos afloramentos rochosos do litoral de Salvador.

\section{MATERIAL E MÉTODOS}

O local escolhido para conduzir o trabalho foi a Praia da Pituba, na orla da cidade de Salvador, Bahia (1300'25.92'S, 38 27'16.67'O) (Figura 1). Os critérios para a escolha deste local foram: 1 - reincidência de chegada de óleo em grandes quantidades na praia durante o impacto agudo, 2 apresentar poríferos e cnidários em abundância e, 3 - ser uma praia central do litoral de Salvador. 


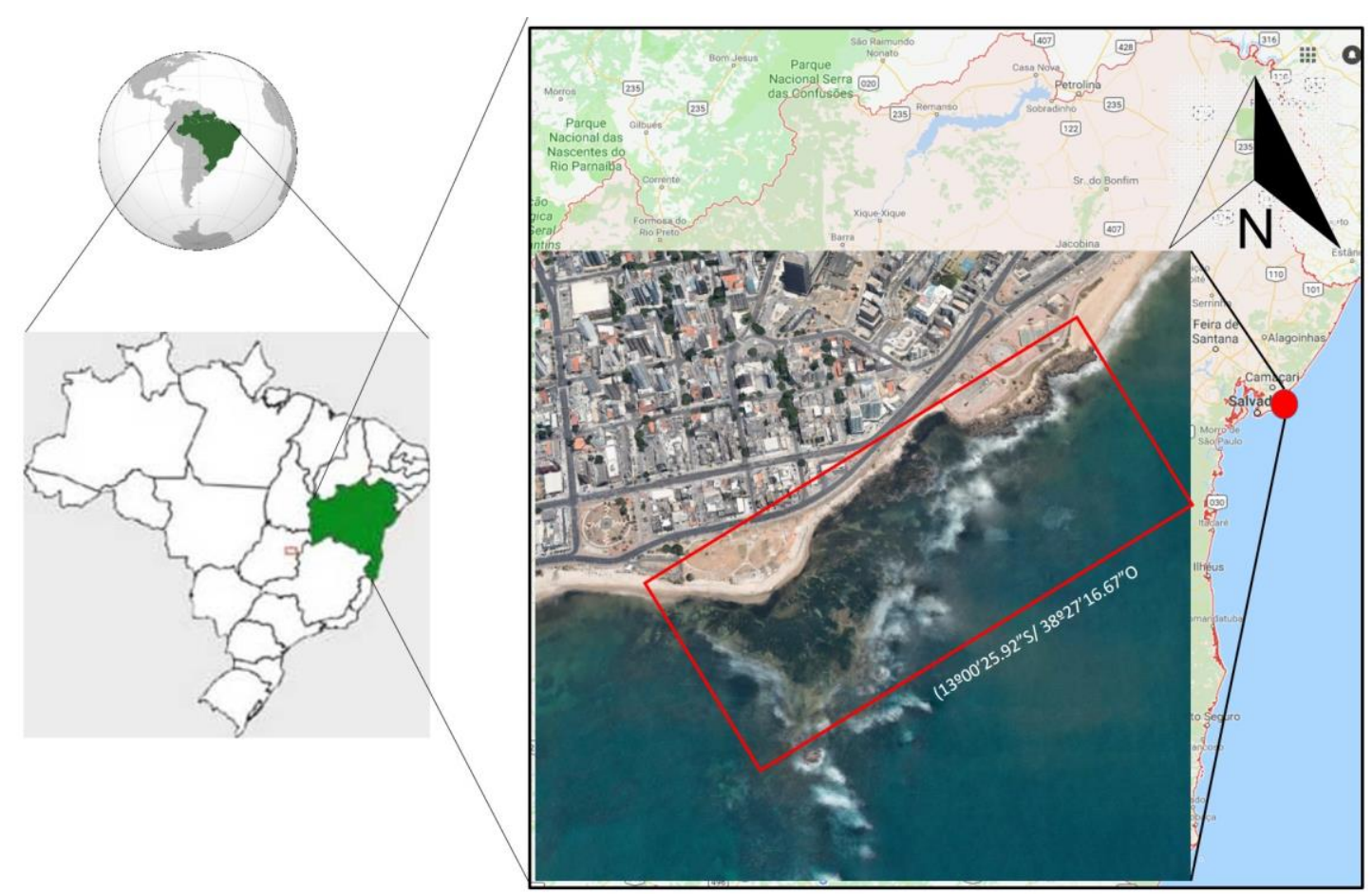

Figura 1: Localização da área de estudo (imagens retiradas e modificadas do Google Maps e Google Earth).

As atividades de campo foram efetivadas durante a maré baixa do dia 09 de novembro de 2019, na zona entre marés, três dias após a limpeza da praia depois da segunda reincidência de óleo no local. Foi realizada uma varredura na faixa de areia e nos afloramentos rochosos em busca de vestígios de petróleo. O tempo de esforço amostral foi de cerca de duas horas. Cada membro da equipe de trabalho percorreu distâncias e caminhos distintos dentro do perímetro apresentado na figura 1, não tendo sido mensurada a distância percorrida. Os poríferos e cnidários encontrados nos afloramentos foram examinados no próprio campo para verificar se existia óleo externamente ou não nos organismos, e independente da ocorrência de óleo, os mesmos foram coletados manualmente, fixados e conservados em solução de formol a 4\%, para analises em laboratório em busca de vestígios de petróleo no interior dos espécimes.

Em laboratório, os exemplares foram identificados utilizando guias e chaves de identificação específicas [11, 12, 13, 14, 15]. Para confirmar o status taxonômico das espécies realizou-se uma consulta ao World Register of Marine Species - WoRMS [16]. Após os procedimentos taxonômicos, os espécimes passaram por necropsia sob microscópio estereoscópico com aumentos de 2 e 4 vezes para observar com mais detalhes a ocorrência de pequenas pelotas de óleo não visualizadas em campo, principalmente nas estruturas internas. Os indivíduos (anêmonas do mar), amostras (esponjas) ou colônias (cnidários coloniais), foram dissecados usando pinças e estiletes e as manchas de petróleo foram identificadas de acordo com a sua textura, viscosidade e brilhos característicos, comparando o material encontrado dentro ou sobre os organismos com amostras de petróleo bruto coletados em campo.

\section{RESULTADOS}

Foram encontrados na praia diversos resquícios de petróleo, tanto na faixa de areia quanto nos afloramentos rochosos e em algumas algas. Foi possível observar também vestígios de óleo mais antigos, intemperizados, os quais apresentavam coloração mais fosca e desgastada, e de óleo mais recente, com uma coloração negra intensa e brilhante. O tamanho das manchas foi bastante variado, indo de pequenas pelotas encontradas na areia a grandes manchas aderidas às superfícies e fendas das rochas (Figura 2). 

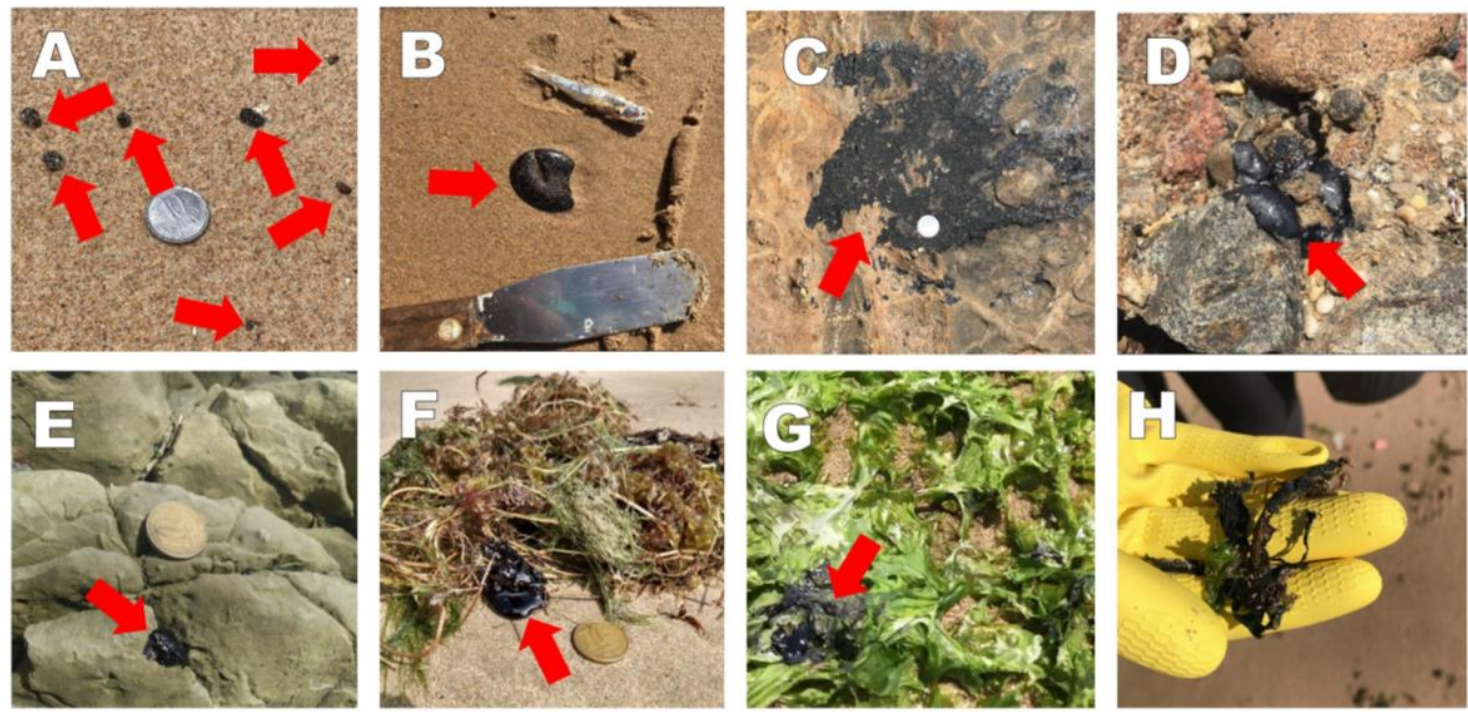

Figura 2: Registros do impacto agudo na Praia da Pituba em Salvador, BA, no dia 09 de novembro de 2019: A - pequenas pelotas na areia; $B$ - fragmento de cerca de $5 \mathrm{~cm}$ de diâmetro; $C$ - mancha grande incrustada em superfície de rocha; $D$ e $E$-manchas de diferentes dimensões entre fendas de rochas. $F, G$ e $H$ - óleo encontrado contaminando algas. Fontes: autores.

No que concerne à fauna, foram identificadas quatro espécies de Porifera e quatro de Cnidaria distribuídas nas seguintes categorias taxonômicas:

Filo Porifera

Classe Demospongiae

Ordem Haplosclerida

Família Niphatidae van Soest, 1980

Gênero Amphimedon Duchassaing \& Michelotti, 1864

Espécie Amphimedon viridis Duchassaing \& Michelotti, 1864

Ordem Tetractinellida

Família Tetillidae Sollas, 1886

Gênero Cinachyrella Wilson, 1925

Espécie Cinachyrella apion (Uliczka, 1929)

Ordem Clionaida

Família Clionaidae d'Orbigny, 1851

Gênero Cliona Grant, 1826

Ordem Poecilosclerida

Espécie Cliona varians (Duchassaing \& Michelotti, 1864)

Família Desmacididae Schmidt, 1870

Gênero Desmapsamma Burton, 1934

Espécie Desmapsamma anchorata (Carter, 1882)

Filo Cnidaria

Classe Anthozoa

Ordem Actiniaria

Família Aiptasiidae Carlgren, 1924

Gênero Bellactis Dube, 1983

Espécie Bellactis ilkalyseae Dube, 1983

Família Actiniidae Rafinesque, 1815

Gênero Bunodosoma Verrill, 1899

Espécie Bunodosoma cangicum Belém e Preslercravo, 1973

Ordem Zoantharia

Família Sphenopidae Hertwig, 1882

Gênero Palythoa Lamouroux, 1816

Espécie Palythoa caribaeorum Duchassaing \& Michelotti, 1860 
Família Zoanthidae Rafinesque, 1815

Gênero Zoanthus Lamarck, 1801

Espécie Zoanthus sociatus (Ellis, 1768)

Durante os trabalhos de campo não foram observados vestígios de óleo contaminando superficialmente os exemplares de poríferos e cnidários coletados, como foi visto em algumas algas (Figura 2), contudo, durante a necropsia em laboratório das esponjas e cnidários coletados foram encontradas pelotas de petróleo na espongiocele de duas das nove amostras de $D$. anchorata (Figura 3A) e na faringe de um dos quatro indivíduos de B. ilkalyseae (Figura 3B). Na Tabela 1 são apresentados o total de amostras/indivíduos/colônias que foram analisados, indicando a presença ou não de vestígios de petróleo observados durante a necrópsia. Estes registros aumentam a quantidade de espécies de invertebrados marinhos bentônicos contaminadas em função do impacto agudo do derramamento de óleo no Nordeste do Brasil no ano de 2019.
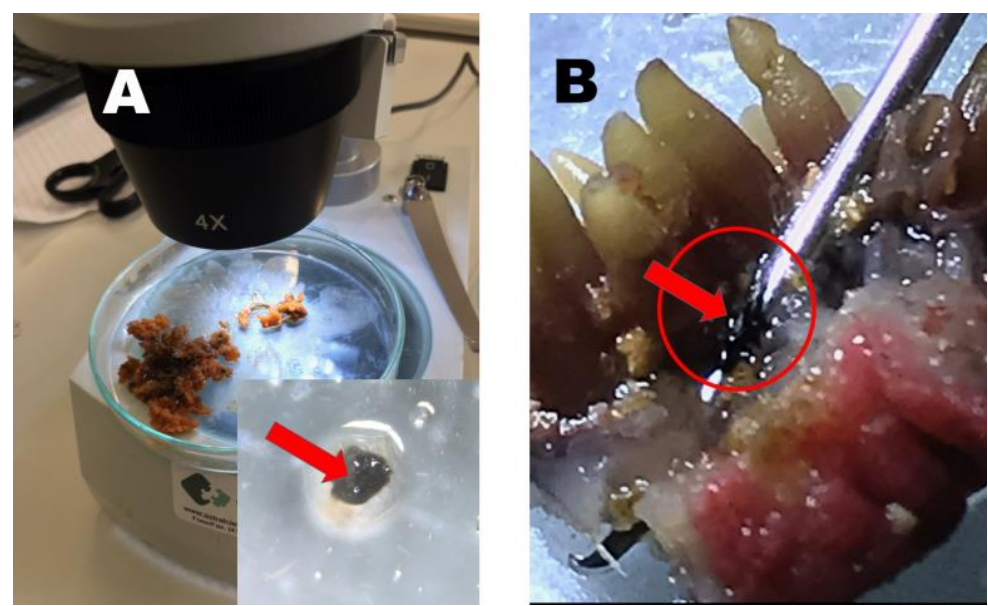

Figura 3: Petróleo na espongiocele da esponja Desmapsamma anchorata $(A)$ e na faringe de Bellactis ilkalyseae (B). Fontes: Walter Cerqueira.

Tabela 1: Ocorrência de petróleo nas espécies coletadas na Praia da Pituba, Salvador (Ba) em novembro de 2019, após reincidência de derramamento oriundo do impacto agudo no litoral nordestino.

\begin{tabular}{|c|c|c|c|}
\hline \multirow[b]{2}{*}{ Espécies } & \multicolumn{3}{|c|}{ Ocorrência de Petróleo } \\
\hline & Total analisado & Presença de óleo & $\%$ \\
\hline \multicolumn{4}{|l|}{ PORIFERA } \\
\hline Amphimedon viridis & 9 amostras & 0 & 0 \\
\hline Cinachyrella apion & 5 indivíduos & 0 & 0 \\
\hline Cliona varians & 9 amostras & 0 & 0 \\
\hline $\begin{array}{l}\text { Desmapsamma anchorata } \\
\text { CNIDARIA }\end{array}$ & 9 amostras & 2 & 22 \\
\hline Bellactis ilkalyseae & 4 indivíduos & 1 & 25 \\
\hline Bunodosoma cangicum & 4 indivíduos & 0 & 0 \\
\hline Palythoa caribaeorum & 9 colônias & 0 & 0 \\
\hline Zoanthus sociatus & 9 colônias & 0 & 0 \\
\hline
\end{tabular}

\section{DISCUSSÃO}

A limpeza das praias realizadas no Nordeste brasileiro, incluindo a Praia da Pituba em Salvador, tanto na areia quanto no substrato rochoso (afloramentos rochosos, arenitos de praia e ambientes recifais), consistiu basicamente na remoção manual, que é um dos diversos métodos recomendados por protocolos internacionais para remoção de petróleo em ambientes litorâneos, por ser um método menos agressivo para o meio ambiente e sua biodiversidade quando comparado a outras estratégias para retirada de óleo, tais como a limpeza mecânica que utiliza bombeamento a vácuo na areia e jateamento nas rochas $[17,18,19,20]$. O método da remoção manual se mostra eficiente para a 
retirada de grandes manchas de óleo, contudo, pequenos resíduos, como as pequenas pelotas encontradas na areia e em algas na Praia da Pituba, não são geralmente contemplados com esta metodologia de limpeza, sendo recomendados, neste caso, métodos biotecnológicos de remediação [21, 22], que são métodos ainda onerosos, principalmente considerando a enorme extensão de área impactada pelo derramamento do ano de 2019 na costa nordestina.

Uma estratégia para retirada de petróleo da areia e das rochas de praias impactadas é a limpeza natural, onde a movimentação das marés e ação das ondas removem naturalmente o óleo [23] e, provavelmente essa estratégia faz com que afloramentos rochosos expostos como os existentes na Praia da Pituba sejam considerados pouco sensíveis a derrames, já que o óleo é naturalmente e rapidamente retirado do ambiente em função da hidrodinâmica [23, 24, 25], o que explicaria as poucas placas e pelotas observadas nos afloramentos quando comparados com a areia da Praia da Pituba, e também o porquê de não ter sido visualizado óleo na superfície de nenhuma das esponjas e cnidários coletados para necropsia em laboratório. Contudo, apesar da literatura [25] informar que a limpeza natural é rápida em ambientes de costões rochosos, o óleo incrustado nas rochas pode demorar meses no ambiente, como constatamos em visita informal à Praia da Pituba (não vinculada à esta pesquisa) em março de 2020, a presença de manchas de óleo intemperizado em várias rochas, similares às encontradas neste trabalho em novembro de 2019 (ver Figura 2C).

O porífero Desmapsamma anchorata apresentou vestígios de óleo na espongiocele e, isto provavelmente ocorre nos organismos desse grupo em função de sua eficiência como filtradores, já que, segundo a literatura [26] $1 \mathrm{~kg}$ de esponjas pode filtrar até $2400 \mathrm{~L}$ de água por hora e ingerir partículas de até $50 \mathrm{~mm}$. Em situações de impacto ambiental as esponjas podem acumular poluentes como HPAs, PCBs, metais traço e radionuclídeos [26, 27, 28, 29] e continuarem vivas, pois muitas espécies são bioacumuladoras [30], explicando o fato de encontrarmos $D$. anchorata com vestígios de petróleo nas cavidades corpóreas internas, mas com aspecto de saudável. Essa espécie é conhecida por ser oportunista e bastante competitiva por espaço, e também é considerada uma espécie insensível a distúrbios ambientais [35]. Alguns estudos também demonstram que essa esponja faz parte da dieta de alguns peixes [13,36, 37].

A anêmona-do-mar Bellactis ilkalyseae apresentou resíduos de óleo na faringe. Ao contrário da esponja $D$. anchorata que é uma espécie filtradora, B. ilkalyseae, assim como outras anêmonas do mar, tem hábito carnívoro [31], podendo o óleo encontrado na faringe deste organismo ter entrado por duas vias: ou através da alimentação, ingerindo uma presa contaminada, ou através dos canais laterais existentes na faringe que são responsáveis pela manutenção da água do esqueleto hidrostático [32]. Este é o primeiro registro de contaminação de petróleo para B. ilkalyseae, até o momento uma espécie endêmica da costa brasileira [33].

Em função da urgência em gerar dados frente ao derramamento de óleo extremamente impactante no litoral nordestino associada à escassez de conhecimentos ecológicos, tornou-se necessário adotar metodologias qualitativas baseadas na observação do ambiente e da fauna, sem tempo para análises mais detalhadas a nível citológico, celular ou molecular dos organismos estudados. Da mesma forma, a situação não permitiu a elaboração de um desenho amostral detalhado e análises estatísticas mais refinadas, optando-se por realizar uma avaliação ecológica rápida com caráter descritivo. Por conta disso, não é possível afirmar que os demais poríferos e cnidários abordados neste trabalho, nos quais não foram registrados vestígios de óleo externa e internamente, não tenham sofrido com o impacto do ano de 2019.

\section{CONCLUSÃO}

Apesar do esforço coletivo de voluntários e governo para limpar as praias, tal limpeza se mostrou eficiente para retirar grandes quantidades de óleo, contudo, muitos resquícios pequenos ou difíceis de remover continuam nas praias contaminando os organismos na costa do Nordeste. O presente trabalho amplia o número de taxa afetados pelo impacto agudo de derramamento de óleo no Nordeste do Brasil no ano de 2019 e registra pela primeira vez a contaminação por petróleo em uma espécie endêmica de anêmona-do-mar e mais um registro para uma espécie de esponja. 


\section{AGRADECIMENTOS}

Aos revisores do artigo pelas valorosas críticas e sugestões, bem como pelo envio de referências para enriquecer a introdução e discussão dos resultados.

\section{REFERÊNCIAS BIBLIOGRÁFICAS}

1. Nadeau RJ, Bergquist ET. Effects of the march 18, 1973 oil spill near Cabo Rojo, Puerto Rico on tropical marine communities. Int Oil Spill Conference, Proceed. 1977 March;1977(1):535-538.

2. Honda M, Suzuki N. Toxicities of Polycyclic Aromatic Hydrocarbons for Aquatic Animals. Int J Environ Res Public Health. 2000;17(1363):1-23, doi:10.3390/ijerph17041363

3. Blackburn M, Mazzacano CAS, Fallon C, Black, SH. Oil in Our Oceans. A Review of the Impacts of Oil Spills on Marine Invertebrates. Portland, OR: The Xerces Society for Invertebrate Conservation. 2014. $152 \mathrm{p}$.

4. Vad J, Kazanidis G, Henry LA, Jones DOB, Tendal OS, Christiansen S, Henry TB, Roberts JM. Potential Impacts of Offshore Oil and Gas Activities on Deep-Sea Sponges and the Habitats They Form. Adv Mar Biol. 2018 Mar;79:33-60. doi: 10.1016/bs.amb.2018.01.001.

5. Ober HK. Effects of Oil Spills on Marine and Coastal Wildlife. Ifas Exension. University of Florida. 2019;2-4. https://edis.ifas.ufl.edu/topic_a47749570

6. Silva JF, Pérez CD. Ecologia trófica das anêmonas-do-mar Anthopleura cascaia e Anthopleura krebsi (Cnidaria: Anthozoa) em duas praias de Pernambuco, Brasil. [Dissertação]. Programa de Pós-Graduação em Biologia Animal, Universidade Federal de Pernambuco, Recife, 2009. https://repositorio.ufpe.br/handle/123456789/581

7. Neves RF, Amaral FD, Steiner AQ. Levantamento de registros dos acidentes com cnidários em algumas praias do litoral de Pernambuco (Brasil). Ciênc Saúde coletiva. 2007 Mar;12(1):231-237, doi:10.1590/S1413-81232007000100026.

8. Tarrant AM, Reitzel AM, Kwok CK, Jenny MJ. Activation of the cnidarian oxidative stress response by ultraviolet radiation, polycyclic aromatic hydrocarbons and crude oil. J Exp Biol. 2014;217:1444-1453, doi: $10.1242 /$ jeb.093690

9. Pena PGL, Northcross AL, Lima MAG, Rêgo RCF. Derramamento de óleo bruto na costa brasileira em 2019: emergência em saúde pública em questão. Cad Saúde Pública. 2020;36(2):e00231019, doi: 10.1590/0102-311x00231019.

10. Araújo ME, Ramalho CWN, Melo PW. Pescadores artesanais, consumidores e meio ambiente: consequências imediatas do vazamento de petróleo no Estado de Pernambuco, Nordeste do Brasil. Cad Saúde Pública. 2020;36(1):e00230319, doi:10.1590/0102-311x00230319.

11. Hooper JNA, Van Soest RWM (Eds.). Systema Porifera: a guide to the classification of Sponges. Kluwer Academic/ Plenum Publishers: New York, Boston, Dordrecht, London, Moscow. 2002, 2 vols. 1706 p.

12. Hooper JNA, Van Soest RWM, Debrenne F. Phylum Porifera Grant, 1826. p. 9-14 in: Hooper, JNA, Van Soest RWM. (Eds.). Systema Porifera: a guide to the classification of sponges. Volume 1. 2002

13. Hajdu E, Peixinho S, Fernandez JCC. Esponjas marinhas da Bahia: guia de campo e laboratório. 2011. Museu Nacional. Rio de Janeiro, $276 \mathrm{p}$.

14. Cairns SD, Gershwin L, Brook FJ, Pugh P, Dawson EW, Ocaña OV, Vervoort W, Williams G, Watson JE, Opresko DM, Schuchert P, Hine PM, Gordon DP, Campbell HJ, Wright AJ, Sánchez JA, Fautin DG. Phylum Cnidaria: corals, medusae, hydroids, myxozoans. in: Gordon, D.P. (Ed.) New Zealand inventory of biodiversity: 1. Kingdom Animalia: Radiata, Lophotrochozoa, Deuterostomia. 2009; 59-101

15. Dube VM. Contribuição ao estudo de anêmonas-do-mar do estado da Bahia. Natura. 1983;83:82-93.

16. WoRMS. World Register of Marine Species. 2020. http://www.marinespecies.org/index.php

17. IMO. Manual on Oil Pollution - Section IV: Combating Oil Spills, Edition (KA569E) (eBook). 2005

18. Harrison RM. Pollution, Causes, Effects and Control, 5 ed. Cambridge, 2014.The Royal Society of Chemistry, e-book.

19. International Tanker Owners Pollution Federation - ITOPF. Apresenta informações, publicações, conselhos e assistência técnica com relação a respostas a poluição por vazamentos de óleo. 2020, Acessível em http://www.itopf.com.

20. Michel J, Shigenaka G, Hoff R. Oil Spill Response and Clean up Techniques. 1992, p. 1-103. In: An Introduction to Coastal Habitats and Biological Resources for Oil Spill Response.NOAA, Seattle, 1992. $401 \mathrm{p}$.

21. Tonini RMCW, Rezende CE, Grativol AD. Degradação e biorremediação de compostos do Petróleo por Bactérias: Uma Revisão. Oecol Australis. 2010;14(4):1021-1035, doi: 10.4257/oeco.2010.1404.12.

22. Vidalli M. Bioremediation: an overview. Pure Appl Chem. 2001;73:1163-1172. 
23. International Petroleum Industry Environmental Conservation Association IPIECA. Biological Impacts of Oil Pollution: Sedimentary Shores. v. 9. 2000. Acessível em http://www.ipieca.org.

24. International Petroleum Industry Environmental Conservation Association IPIECA. Biological Impacts of Oil Pollution: Sedimentary Shores. v. 7. 1995. Acessível em http://www.ipieca.org.

25. Cantagallo C, Milanelli JCC, Dias-Brito D. Limpeza de ambientes costeiros brasileiros contaminados por petróleo: uma revisão. Pan-American J Aquat Sci. 2007;2(1):1-12.

26. Batista D, Tellini K, Nudi AH, Massone TP, Scofield AL, Wagener ALR. Marine sponges as bioindicators of oil and combustion derived PAH in coastal Waters. Mar Environ Res. 2013;92:234-243, doi:10.1016/j.marenvres.2013.09.022

27. Patel B, Balani MC, Patel S. Sponge 'sentinel' of heavy metals. Sci total environ. 1985;41(2):143-152, doi:10.1016/0048-9697(85)90184-6

28. Mestre C, Maher W, Roberts D, Broad A, Krikowa F, Davis AR. Sponges as sentinels: Patterns of spatial and intra-individual variation in trace metal concentration. Mar Pollut Bull. 2012;64(1):80-89, doi:10.1016/j.marpolbul.2011.10.020

29. Perez T, Wafo E, Fourt M, Vacelet J. Marine Sponges as Biomonitor of Polychlorobiphenyl Contamination: Concentration and Fate of 24 Congeners. Environ Sci Technol. 2003;37(10):2152-2158, doi:10.1021/es026234v

30. Esteves AIS, Esponjas Marinhas: Potenciais Aplicaç̃es Biotecnológicas. [Tese] Faculdade de Ciências, Universidade de Lisboa, Portugal. 2009. 298 p.

31. Nagai Y, Nagai S. Feeding factors for the sea anemone Anthopleura midorii. Mar Biol. 1973;18:55-60, doi:10.1007/BF00347921

32. Zamponi MO. El sistema mesoendoesqueletal en anémonas de mar (Cnidaria, Hexacorallia, Actiniaria). Rev Real Acad Galega Cien. 2007;26:43-52.

33. Oliveira TGL, Gomes PB. Primeira descrição do comportamento de Bellactis ilkalyseae (Cnidaria: Actiniaria) durante o processo de reprodução assexuada. Trop Oceanogr. 2005;33(1):67-72, doi: 10.5914/tropocean.v33i1.5071

34. Dunlap M, Pawlik JR. Video-monitored predation by Caribbean reef fishes on an array of mangrove and reef sponges. Mar Biol. 1996;126:117-123.

35. Aerts LAM. 1999. Sponge-coral interactions on Caribbean reefs. PhD thesis. University of Amsterdam. Cap. 6: Aerts L, Scheffers S, Gomez R. Ecological strategy and competitive ability in the opportunistic reef sponge Desmapsamma anchorata.

36. Lorders FL, Miranda RJ, Nunes, JACC, Barros F. Spongivory by Fishes on Southwestern Atlantic Coral Reefs: No Evidence of Top-Down Control on Sponge Assemblages. Front Mar Sci. 2018;5:1-9. doi:10.3389/fmars.2018.00256.

37. Wulff JL. Ecological interactions of marine sponges. Can J Zool. 2006;84:146-166. 